\title{
Structure and antioxidant activity of a novel polysaccharide derived from Amanita caesarea
}

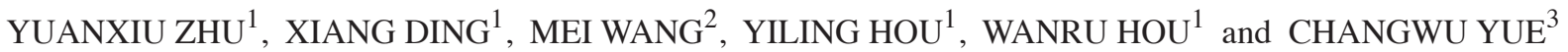 \\ ${ }^{1}$ Key Laboratory of Southwest China Wildlife Resources Conservation (Ministry of Education), \\ College of Life Sciences, China West Normal University, Nanchong, Sichuan 637009; \\ ${ }^{2}$ National Center for Sweet Potato Improvement Centre of Nanchong, Nanchong Academy of Agricultural Sciences, \\ Nanchong, Sichuan 637001; ${ }^{3}$ Key Laboratory of Characteristic Microbial Resources \& Drug Development of \\ Guizhou Provincial Education Department, Zunyi Medical University, Zunyi, Guizhou 561000, P.R. China
}

Received July 12, 2015; Accepted August 10, 2016

DOI: $10.3892 / \mathrm{mmr} .2016 .5693$

\begin{abstract}
A heteropolysaccharide was isolated from the fruiting bodies of Amanita caesarea using a diethylaminoethyl-cellulose column, Sephacryl S-300 gel column and Sephadex G-200 column. The Amanita caesarea polysaccharide was predominantly composed of $\alpha$-D-glucose and $\alpha$-D-lyxose at a ratio of $2: 1$, and it had a molecular weight of 19,329 Da. The structural features of the Amanita caesarea polysaccharide were investigated by a combination of total hydrolysis, methylation analysis, gas chromatography-mass spectrometry, and infrared spectra and nuclear magnetic resonance spectroscopy. The results showed that Amanita caesarea polysaccharide (termed AC-1) had a backbone of 1,4-linked $\alpha$-D-glucose and 1,3,6-linked $\alpha$-D-glucose, with branches of one 1-linked $\alpha$-D-lyxose residue. The antioxidant activity of $\mathrm{AC}-1$ was evaluated by two biochemical methods, 2,2-azino-bis diammonium $\left(\mathrm{ABTS}^{+}\right.$) radical scavenging activity and 1,1-diphenyl-2-picrylhydrazyl $\left(\mathrm{DPPH}^{-}\right)$radical scavenging activity. The uncontrolled production of free radicals is involved in various diseases, including cancer, atherosclerosis and degenerative aging processes. The results indicated that the Amanita caesarea polysaccharide exhibits strong antioxidant activity, thus, it may be a useful natural product antioxidant.
\end{abstract}

\section{Introduction}

The uncontrolled production of free radicals is involved in various diseases, including cancer and atherosclerosis, and degenerative aging processes. It is important to develop

Correspondence to: Dr Yiling Hou, Key Laboratory of Southwest China Wildlife Resources Conservation (Ministry of Education), College of Life Sciences, China West Normal University, 1 Shida Road, Nanchong, Sichuan 637009, P.R. China

E-mail: biostart8083@126.com

Key words: structure elucidation, antioxidant activity, polysaccharide, Amanita caesarea effective antioxidants with low toxicity that protect the human body from free radicals and, thus, a number of chronic diseases. Polysaccharides consist of polymeric structures composed of at least ten monosaccharides sequentially connected by glycosidic bonds. Polysaccharides can be classified as homopolymers, a term used to indicate a polymer composed of identical monosaccharides, or heteropolymers, a term used to classify polysaccharides composed of two or more types of monosaccharides. Fungal polysaccharides are a type of active organic compound that are found in the fruiting bodies, mycelium and fermentation broth of large edible and medicinal fungi $(1,2)$. A large number of studies have demonstrated that fungal polysaccharides exhibit a variety of biological activities, including anti-aging, antitumor, anti-oxidation and immunoregulatory activities, and are safe with low toxicity (3-10).

Amanita caesarea is a type of fungi of the Amanita genus, which grows in the Garzê county of Sichuan province (China) at an elevation of $3,800 \mathrm{~m}$. In this study, a water-soluble polysaccharide (termed AC-1) was obtained from the fruiting bodies of Amanita caesarea using a diethylaminoethyl (DEAE)-cellulose column, Sephacryl S-300 gel column and Sephadex G-200 column. To the best of our knowledge, its chemical structure was characterized for the first time in the present study. Structural analysis of the fraction was conducted using chemical methods, infrared spectra spectroscopy and nuclear magnetic resonance spectroscopy. The antioxidant activity of AC-1 was also evaluated by two antioxidant assays. The result of this study introduced Amanita caesarea as a possible valuable natural product, which exhibited unique antioxidant properties.

\section{Materials and methods}

Materials. Fresh Amanita caesarea was collected in the Garzê county of Sichuan province, for which specific permission was not required as it is an open village in China. The field studies did not involve endangered or protected species as the endangered or protected species protection zone was not entered for sampling. Following vacuum freeze-drying, the Amanita caesarea were crushed and stored at $4^{\circ} \mathrm{C}$ prior 
to use in the Key Laboratory of Southwest China wild Resources Conservation (Ministry of Education), College of Life Sciences, China West Normal University (Nanchong, China). Trifluoroacetic acid (TFA), standard monosaccharides and dextrans of different molecular weights were purchased from Beijing Biodee Biotechnology Co., Ltd. (Beijing, China). DEAE-cellulose column, Sephacryl S-300 gel column and Sephadex G-200 column were purchased from Sigma-Aldrich; Merck Millipore (Darmstadt, Germany). All other reagents used were of analytical grade.

Extraction, isolation and purification of polysaccharides from the fruiting bodies of Amanita caesarea. The fresh Amanita caesarea was thoroughly washed with water, dried at $60^{\circ} \mathrm{C}$, and then powdered with a pulverizer. For conventional extraction, dried and powdered Amanita caesarea (200 g) was accurately weighed and extracted with 2,000 $\mathrm{ml}$ distilled water at $85^{\circ} \mathrm{C}$ for $6 \mathrm{~h}$. The extract was filtrated and centrifuged at $17,925 \mathrm{x} g$ for $20 \mathrm{~min}$ in a high-speed centrifuge and concentrated in a vacuum. Then the supernatant was added to $6 \mathrm{X}$ 95\% EtOH to precipitate the crude polysaccharides. After the deproteination as described previously (11), the crude polysaccharides were redissolved in $100 \mathrm{ml}$ distilled water, purified with the Sephacryl S-300 gel column and DEAE-cellulose column according to the manufacturer's instructions. The polysaccharides were eluted stepwise, fractionated with 0.1 , $0.2,0.3,0.4$ and $0.5 \mathrm{~mol} / 1 \mathrm{NaCl}$ and monitored using the phenol-sulfuric acid method (12). The $0.1 \mathrm{M} \mathrm{NaCl}$ elution was purified, concentrated using the Sephadex G-200 column, passed through a $6-\mathrm{kDa}$ membrane for $36 \mathrm{~h}$ to eliminate small molecular compounds and lyophilized overnight in a Christ Alpha 1-2 LD freeze dryer (Martin Christ Gefriertrocknungsanlagen $\mathrm{GmbH}$, Osterode am Harz, Germany). AC-1 were obtained by vacuum freeze drying for further analysis of their structure.

Determination of the molecular weight of AC-1. The molecular weight of the polysaccharide fraction was identified by high-performance gel permeation chromatography (HPGPC) as described previously (13). Briefly, an aliquot (5 mg) of the dry polysaccharide was dissolved in $5 \mathrm{ml}$ double-distilled water and filtered through a membrane filter $(0.22 \mu \mathrm{m})$. The calibration curve was prepared from the standard T-series Dextran. The data were analyzed using GPC software (Millennium 32 software; Agilent Technologies, Inc., Santa Clara, CA, USA).

Monosaccharide composition analysis of AC-1. The polysaccharide AC-1 (5 mg) was hydrolyzed with $2 \mathrm{M}$ TFA at $110^{\circ} \mathrm{C}$ for $6 \mathrm{~h}$ using acid-catalyzed hydrolysis (14). When hydrolysis was completed, the products were dissolved with distilled water for monosaccharide composition analysis. Monosaccharide composition was measured by a high-performance liquid chromatography (HPLC) refractive index detector (Agilent 1100 series; Agilent Technologies, Inc.). The HPLC was performed under the following conditions: A concentration of refined polysaccharide of $20 \mathrm{mg} / \mathrm{ml}, 75 \%$ acetonitrile as the mobile phase at $1.4 \mathrm{ml} / \mathrm{min}$ and a column oven temperature of $35^{\circ} \mathrm{C}$ (15). D-glucose, D-mannose, D-fructose, D-galactose, L-rhamnose, L-arabinose and D-xylose were used as standard sugars.
Fourier transform-infrared spectra (FT-IR) analysis. FT-IR spectra of the polysaccharide AC-1 were measured by grinding a mixture of polysaccharide with dry $\mathrm{KBr}$, then pressing into pellets. FT-IR spectra of the AC-1 was collected using a Thermo Nicolet 6700 spectrometer (Thermo Fisher Scientific Inc., Waltham, MA, USA) operating in the range of $400-4,000 \mathrm{~cm}^{-1}$ at a resolution of $2 \mathrm{~cm}^{-1}$.

Methylation analysis. The polysaccharide was methylated using methyliodide as described previously (16). The completeness of methylation was confirmed by the disappearance of the hydroxyl absorption in IR spectrum at $3,400 \mathrm{~cm}^{-1}$. The permethylated product was depolymerized with $90 \%$ formic acid at $100^{\circ} \mathrm{C}$ for $4 \mathrm{~h}$, and further hydrolyzed with $2 \mathrm{M}$ TFA at $100^{\circ} \mathrm{C}$ for $6 \mathrm{~h}$. The resulting products were derivatized using the derivatization reagent and analyzed by gas chromatography-mass spectrometry (GC-MS) as described previously (17).

Nuclear magnetic resonance (NMR) analysis. The polysaccharide was dissolved in deuteroxide accompanied by ultrasonic wave precessing for $10 \mathrm{~min}$. Ultrasonic wave precessing was conducted using a Bransonic CPX-3800H (Branson Ultrasonics, Danbury, CT, USA) and precessing increases the dissolution rate of polysaccharides. Then the Varian Unity INOVA 400/45 (Agilent Technologies, Inc.) was used to perform the ${ }^{13} \mathrm{C}$ NMR spectra and ${ }^{1} \mathrm{H}$ NMR spectra analyses with tetramethylsilane as internal standard.

$D P P H^{-}$radical scavenging activity. The $\mathrm{DPPH}^{-}$radical scavenging activity of the polysaccharide sample was measured by a decrease in absorbance at $517 \mathrm{~nm}$ of a solution of purple-colored $\mathrm{DPPH}^{-}$in methanol brought about by the sample (18). A lower absorbance of the reaction mixture indicates higher free radical scavenging activity. Absorbance at $517 \mathrm{~nm}$ was measured after $30 \mathrm{~min}$ using a UV-visible spectrometer. The capability to scavenge the $\mathrm{DPPH}^{-}$radical was calculated using the following equation:

$$
\text { DPPH scavenge }(\%)=\left(\frac{1-A_{t e s t}}{A_{\text {control }}}\right) \times 100
$$

Where $\mathrm{A}_{\text {control }}$ is the absorbance of the control reaction and $\mathrm{A}_{\text {test }}$ is the absorbance in the presence of the polysaccharide sample (19). The antioxidant activity of the extract was compared with that of vitamin $\mathrm{C}(\mathrm{Vc})$ butylated hydroxytoluene (BHT).

ABTS radical scavenging activity. $\mathrm{ABTS}^{+}$radical scavenging activity of the polysaccharide extracts and fractions was measured using the $\mathrm{ABTS}^{+}$cation decolorization assay as described previously (20). Briefly, the $\mathrm{ABTS}^{+}$radical cation was produced by reaction of $7 \mathrm{mM}$ stock solution $\mathrm{ABTS}^{+}$with $2.45 \mathrm{mM}$ ammonium persulphate and then the mixture was incubated at room temperature in the dark for $16 \mathrm{~h}$. Then $2 \mathrm{ml}$ of various concentrations of the sample and $2 \mathrm{ml}$ of $0.7 \mathrm{mM}$ $\mathrm{ABTS}^{+}$radical solution were added. A control reaction was conducted without the polysaccharide extracts. The absorbance was measured immediately at $734 \mathrm{~nm}$. The percentage of scavenging of hydrogen radicals was calculated as follows:

$$
\text { Scavenging effect }(\%)=\left[1-\frac{\left(A_{\text {sample }}-A_{\text {sample }+ \text { blank }}\right)}{A_{\text {control }}}\right] \times 100
$$


Table I. ${ }^{13} \mathrm{C}$ nuclear magnetic resonance chemical shift data $(\delta, \mathrm{ppm})$ for polysaccharide AC-1

\begin{tabular}{|c|c|c|c|c|c|c|}
\hline \multirow[b]{2}{*}{ Sugar residue } & \multicolumn{6}{|c|}{ Chemical shift, $\delta$ (ppm) } \\
\hline & $\mathrm{C} 1$ & $\mathrm{C} 2$ & $\mathrm{C} 3$ & $\mathrm{C} 4$ & $\mathrm{C} 5$ & C6 \\
\hline$\rightarrow 4)-\alpha-D-G l c p-(1 \rightarrow$ & 97.92 & 68.32 & 70.85 & 72.63 & 69.56 & 62.56 \\
\hline$\rightarrow 3,6)-\alpha-D-G l c p-(1 \rightarrow$ & 98.26 & 68.87 & 71.03 & 74.86 & 69.90 & 69.90 \\
\hline$\alpha-D-1 y x-(1 \rightarrow$ & 101.50 & 69.51 & 71.78 & 78.01 & 70.44 & 61.08 \\
\hline
\end{tabular}

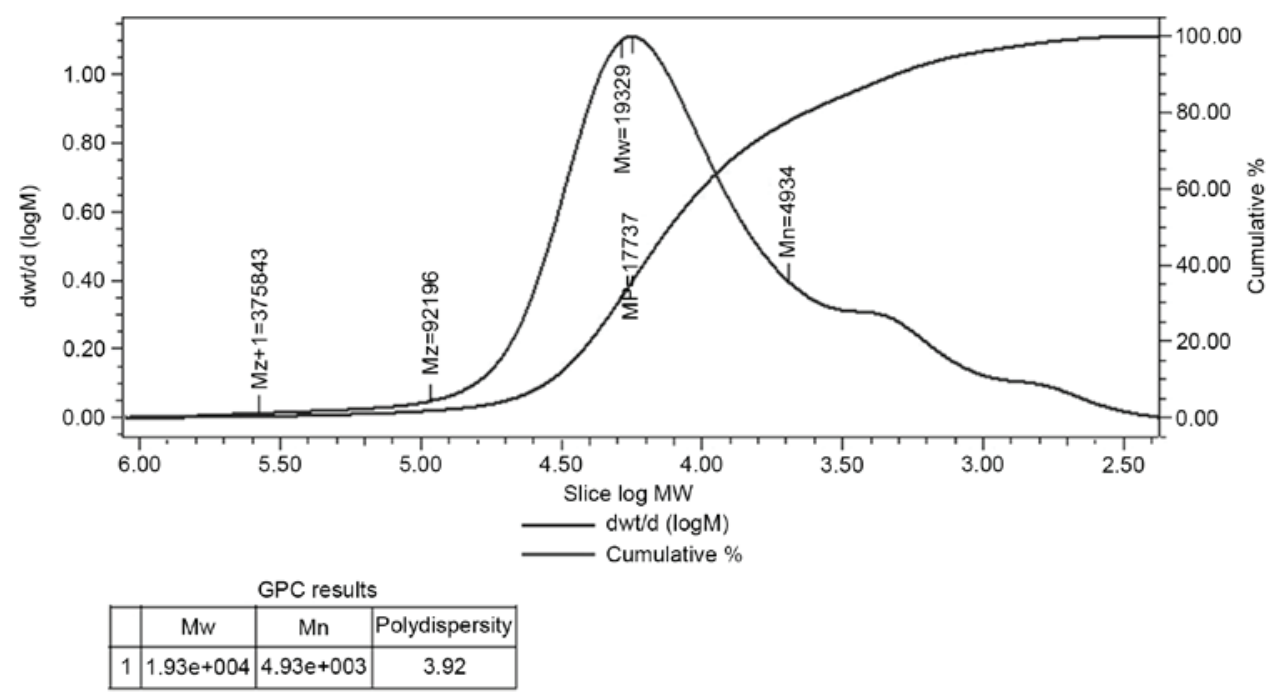

Figure 1. High-performance gel permeation chromatography analysis. The Mw of AC-1 was 19,329 Da, the peak-molecular weight was 17,737 Da, the Mn was $4934 \mathrm{Da}$, and the polydispersity was 3.92. Mw, weight-average molecular weight; Mn, number-average molecular weight.

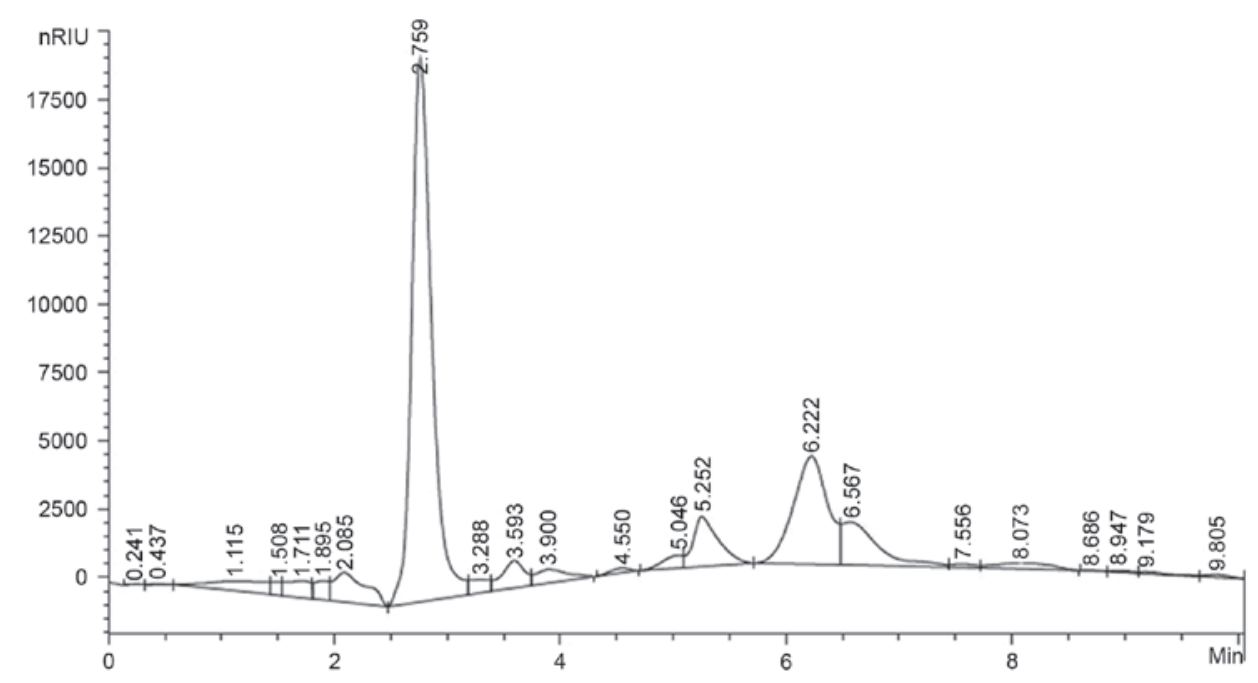

Figure 2. Component monosaccharide analysis of polysaccharides by high performance liquid chromatography.

Where $\mathrm{A}_{\text {control }}$ was the absorbance of the control group in the $\mathrm{ABTS}^{+}$radicals generation system, $\mathrm{A}_{\text {sample }}$ was the absorbance of the test group and $\mathrm{A}_{\text {sample }}$ blank was the absorbance of the samples only. VC was used as a positive control.

Statistical analysis. All data are presented as the mean \pm standard deviation of three replications. Statistical analyses were performed using Student's t-test and one-way analysis of variance with SPSS software (version 20; IBM SPSS, Armonk, NY, USA). P<0.05 was considered to indicate a statistically significant difference.

\section{Results}

Extraction, isolation and purification of polysaccharides. The crude polysaccharide, termed AC-1, was obtained as a 
Table II. Gas chromatography-mass spectrometry results of methylation analysis of AC-1.

\begin{tabular}{lll}
\hline Methylated sugar & Linkage & \multicolumn{1}{c}{$\mathrm{m} / \mathrm{z}$} \\
\hline $2,3,6-\mathrm{Me}_{3}$-Glcp & $1,4-$ & $45,59,73,88,101,113,133,146,159,175,185,201,217,232$ \\
$2,4-\mathrm{Me}_{2}$-Glcp & $1,3,6-$ & $41,59,73,89,101,117,131,146,159,173,191,217,233$ \\
$2,3,4-\mathrm{Me}_{3}$-Lyx & $1-$ & $45,58,73,88,101,115,133,146,159,174,185$ \\
\hline
\end{tabular}

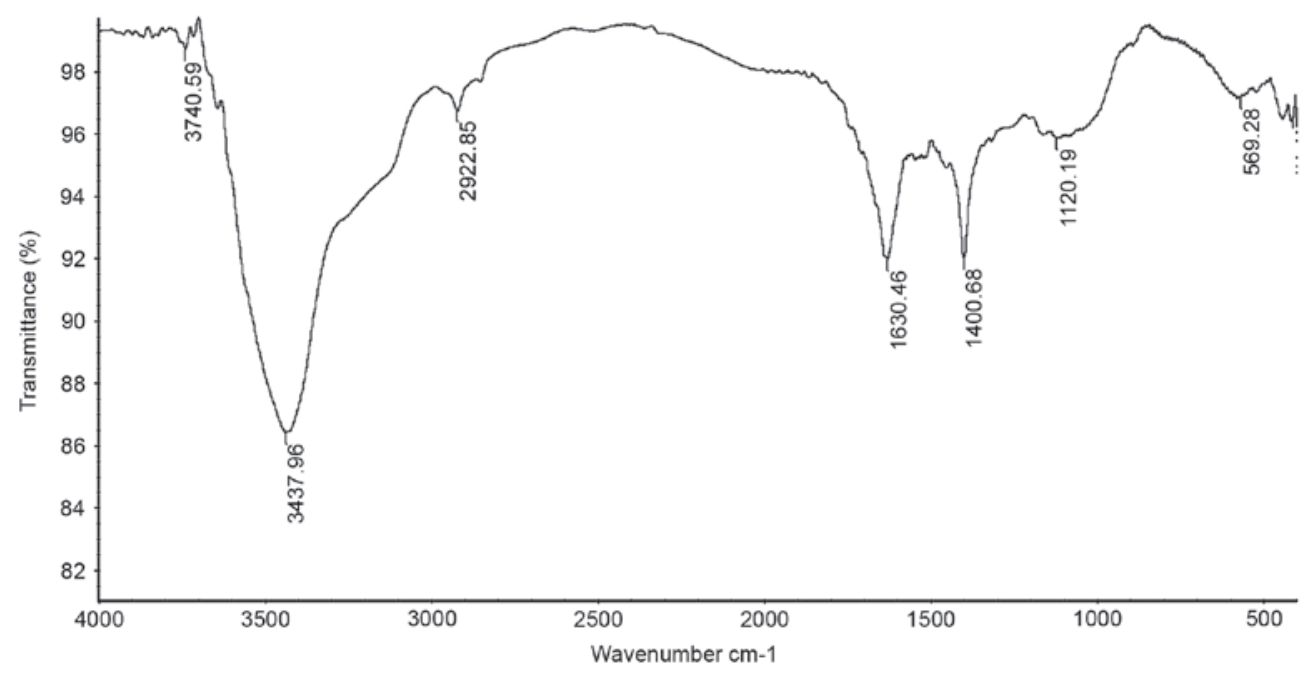

Figure 3. Fourier transform-infrared spectra analysis of AC-1.

water-soluble light yellow powder from the fruiting bodies of Amanita caesarea, with a yield of $10.8 \%$.

Determination of molecular weight. HPGPC of the polysaccharide fraction demonstrated that each fraction was represented by a broad and symmetrical peak on the chromatograms. The dextran standards were used to create a calibration curve for elucidating the molecular weight of AC-1. Weight-average molecular weight of AC-1 was 19,329 Da and the polydispersity was 3.92 (Fig. 1).

Monosaccharide composition analysis. The composition analysis of polysaccharides is an important quality control step to determine basic information regarding the polysaccharides. In this study, the AC-1 polysaccharide samples were hydrolyzed with TFA and then the component monosaccharides were analyzed by HPLC with Agilent refractive index detector. It is shown that the AC-1 polysaccharides were composed of D-Glucose and D-Xylose (Fig. 2).

FT-IR analysis. The IR spectrum of the sample (Fig. 3) showed that the absorption was greatest at $>3,000 \mathrm{~cm}^{-1}$, which was caused by the stretching and angular vibration of the $\mathrm{O}-\mathrm{H}$ linkage. In addition, the absorption peak observed at $2,922.85 \mathrm{~cm}^{-1}$ was due to $\mathrm{C}-\mathrm{H}$ stretching vibration, the absorption peak at $1,630.46 \mathrm{~cm}^{-1}$ was caused by $\mathrm{OH}$ deformation vibration and the strong absorption peaks at $1,400.68 \mathrm{~cm}^{-1}$ were due to $\mathrm{C}-\mathrm{H}$ bending vibration. The absorption peaks at $1,120.19 \mathrm{~cm}^{-1}$ in the range of $1,200-1,000 \mathrm{~cm}^{-1}$ in the IR spectrum suggested that the monosaccharides in the two samples had a pyranose-ring. In addition, the peaks at $569.28 \mathrm{~cm}^{-1}$ were due to $\mathrm{C}-\mathrm{H}$ rocking vibration.
Analysis of the NMR results. The hydrogen spectrum of AC-1 is shown in Fig. 4. In the ${ }^{1} \mathrm{H}$ NMR (400HZ) spectrum, $\delta 5.06$ and $\delta 4.98$ indicate that there were two anomeric hydrogens existing in $\mathrm{AC}-1$. This suggests that $\mathrm{AC}-1$ is composed of two monosaccharides ( $\alpha$ pyranose), as the $\mathrm{H}-1$ chemical shift was $>4.95$. The signals at $\delta 3.647-\delta 4.196$ are the signal peak overlaps of remaining protons. $\delta 4.793$ and $\delta 4.814$ were the hydrogen signals of water. The ${ }^{13} \mathrm{C}$ NMR spectrum of AC-1 (Fig. 5) showed that the anomeric peaks were centralized at $\delta 97.917, \delta 98.258$ and $\delta 101.494 \mathrm{ppm}$, indicating that there was only an $\alpha$-anomeric configuration in $\mathrm{AC}-1$. The results are consistent with the analysis results of IR and ${ }^{1} \mathrm{H}$ NMR. A chemical shift was not observed in the region between $\delta 160$ and $\delta 180 \mathrm{ppm}$, indicating that there was no hyaluronic acid in AC-1. The presence of the AC-1 signal confirmed that all monomers had a pyran ring, as furan ring signals should be around $\delta 107-109 \mathrm{ppm}$. According to the literature, the resonances in the region of 97-101 ppm in the ${ }^{13} \mathrm{C} \mathrm{NMR}(400 \mathrm{MHz})$ spectrum of $\mathrm{AC}-1$ were attributed to the anomeric carbon atoms of -D-lyxopyranose (-D-lyx) and -D-glucosepyranose (-D-Glup). In the anomeric carbon region, the signal at $\$ 97.92$ could be attributed to C-1 of $\rightarrow 4)-\alpha-D-G l u-(1 \rightarrow$; the signal at $\delta 98.26$ to $C-1$ of $\rightarrow 3,6)-\alpha-D-G l u-(1 \rightarrow$; and the signal at $\delta 101.50$ to $\mathrm{C}-1$ of $\alpha$-D-lyx-( $1 \rightarrow$ (Fig. 5). The assignment of the carbon atom signals is shown in Table I.

Methylation analysis. The methylated products of AC-1 were hydrolyzed with acid, converted into alditol acetate, and analyzed by GC-MS. Experimental data are shown in Table II. The information in MS showed that fragment ion peaks were consistent with data of D-configuration monosaccharide 


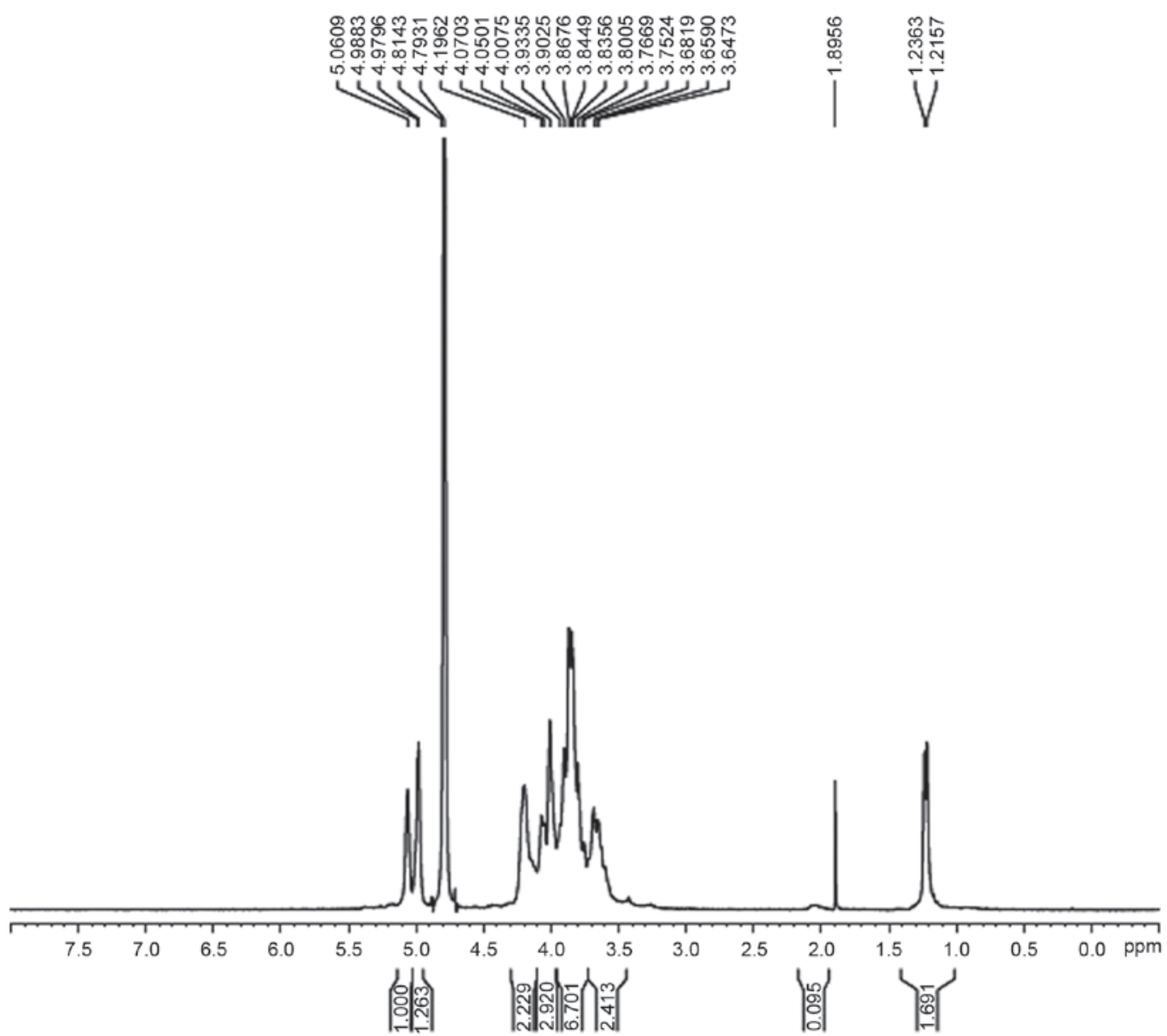

Figure 4. ${ }^{1} \mathrm{H}$ nuclear magnetic resonance spectra analysis of AC-1.
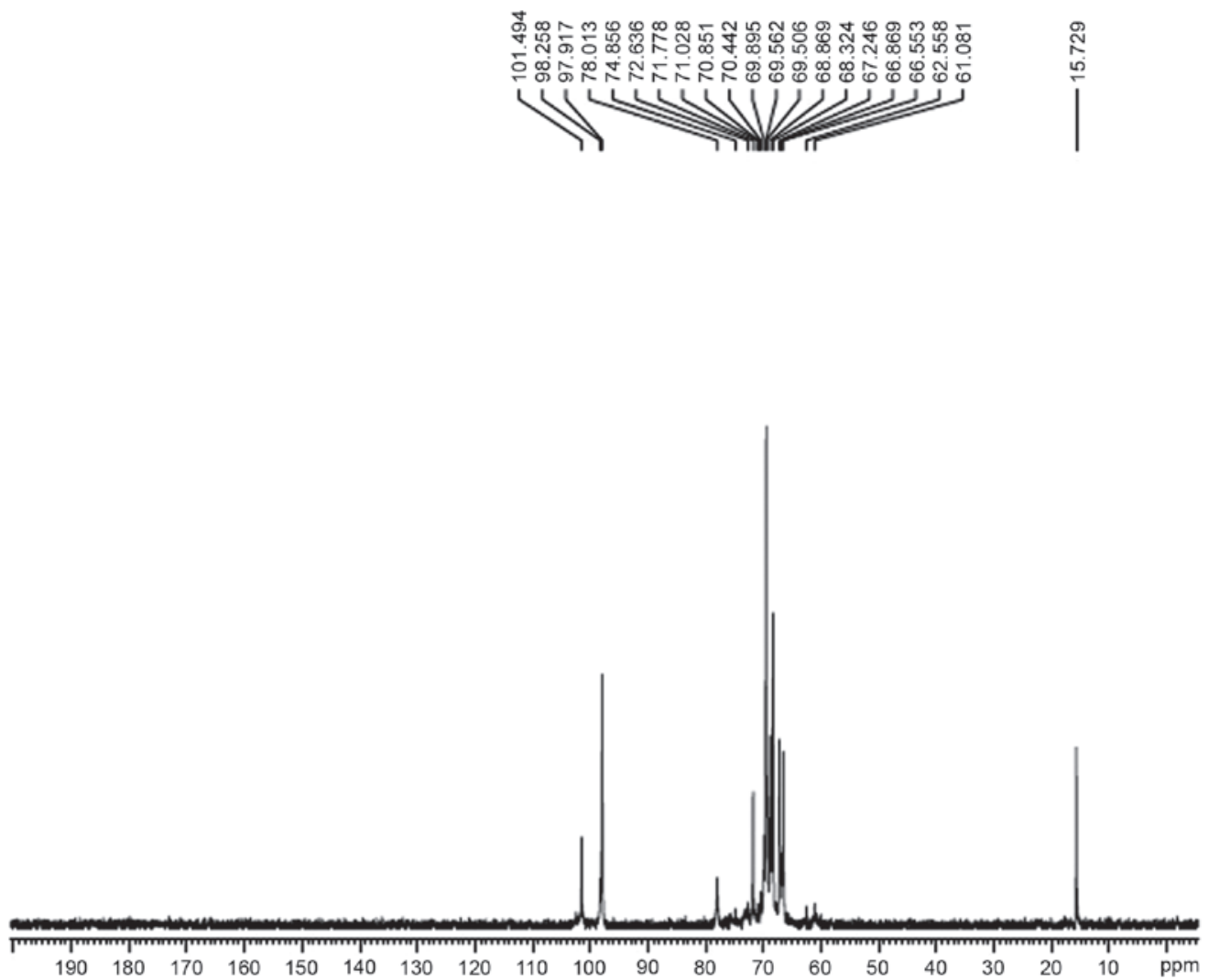

Figure $5 .{ }^{13} \mathrm{C}$ Nuclear magnetic resonance spectra analysis of $\mathrm{AC}-1$. 
A

SI:82 Formula:C15H34O6Si2 CAS:32388-39-9 MolWeight:366 Retlndex:1710

CompName:Glucopyranose, 2,3,6-tri-O-methyl-1,4-bis-O-(trimethylsilyl)-, D-\$\$ 2,3,6-Tri-O-methyl, 1,4-bis-O-(trimethylsily)hexopyranose 100

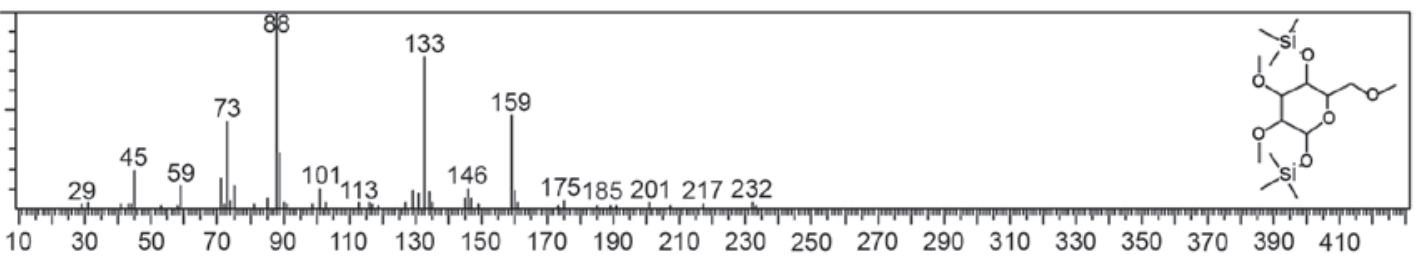

$\mathbf{B}$

SI:69 Formula:C17H4006Si3 CAS:55400-19-6 MolWeight:424 Retlndex:1819

CompName:Glucopyranose, 2,4-di-O-methyl-1,3,6-tris-O-(trimethylsilyl)-\$ 2,4-Di-O-methyl-1,3,6-tris-O-(trimethylsilyl)hexopyranose

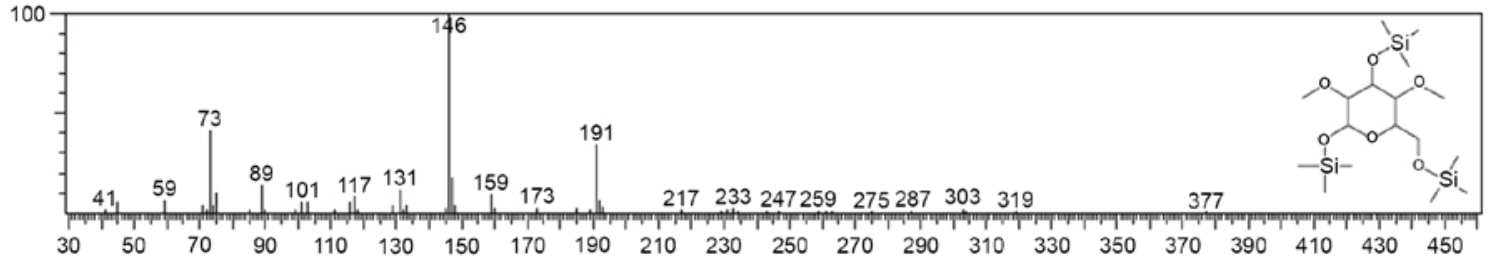

C

SI:69 Formula:C11H240O5Si CAS:20561-82-4 MolWeight:264 Retlndex:1365

CompName:Lyxopyranose, 2,3,4-di-O-methyl-1-O-(trimethylsilyl)-\$ \$2,3,4-Tri-O-methyl-1-O-(trimethylsilyl)hexopyranose \# \$\$

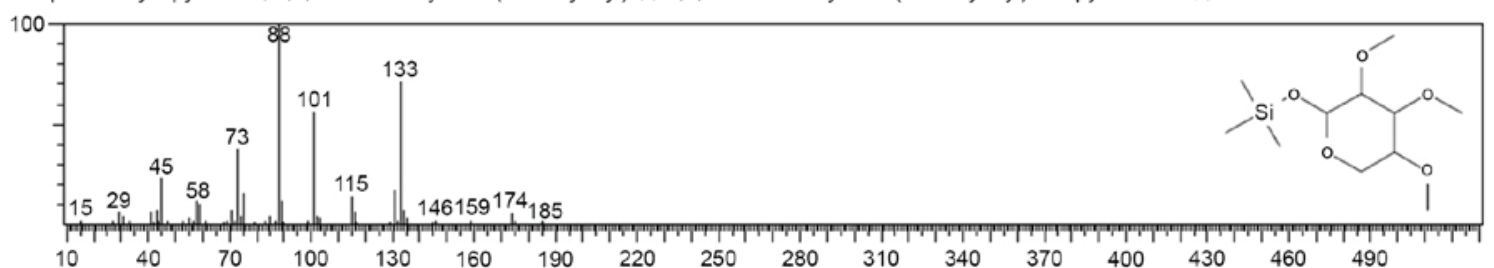

Figure 6. Gas chromatography-mass spectrometry analysis of AC-1. (A) The fragment ion peaks of 2, 3, 6-Me - -Glcp. (B) The fragment ion peaks of 2 , 4-Me - -Glcp. (C) The fragment ion peaks of 2,3,4-Me $-\mathrm{Lyx}$.

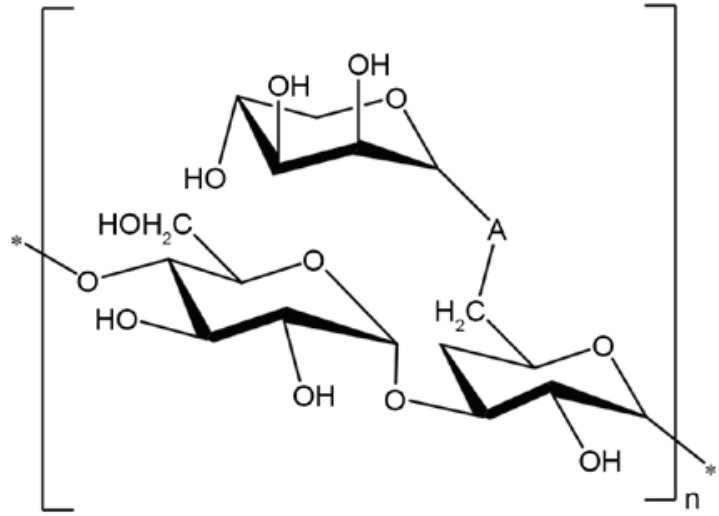

Figure 7. Predicted chemical structure of polysaccharide AC-1.

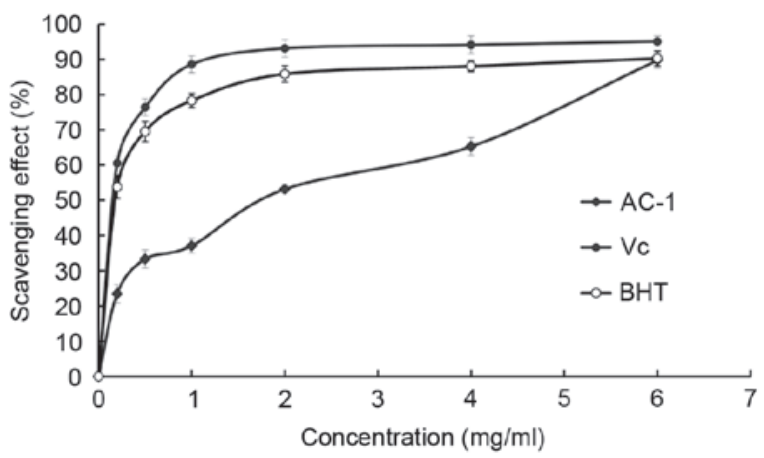

Figure 8. DPPH' radical scavenging effect of AC-1 from Amanita caesarea. Vc, vitamin C.

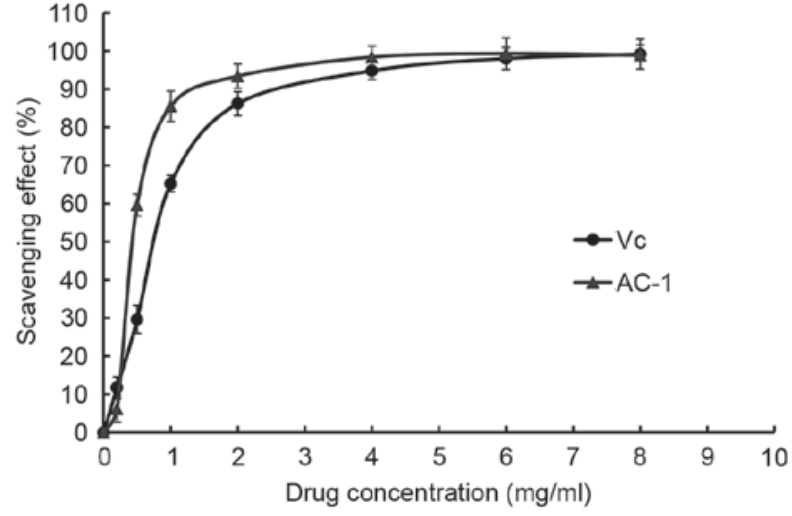

Figure 9. $\mathrm{ABTS}^{+}$radical cation scavenging activity of AC-1. Vc, vitamin C.

fragment ion peaks, which suggested that the glucose and lyxose residues were both of D-configuration. Methylation analysis for AC-1 demonstrated that the $\alpha$-D-glucosepyranose residues were 2,4-bis-substituted and 2,3,6-trisubsituted, and the $\alpha$-D-lyxopyranose residues were 2,3,4-tri-substituted (Fig. 6 and Table II). Results of methylated linkage analysis of AC-1 indicated that $(1 \rightarrow 4)$-linked- $\alpha$-D-glucosepyranose was one of the largest residues of the polysaccharide structure, the branched residue was $(1 \rightarrow 3,6)$-linked- $\alpha$-D-glucosepyranose revealing that $(1 \rightarrow 3)$-linked- $\alpha$-D-glucosepyranose forms the backbone of the structure. Residues of branch structure terminated with $\alpha$-D-lyxopyranose residues. It was concluded that a repeating unit of AC-1 has a backbone of $(1 \rightarrow 4)$ - $\alpha$-D-glucosepyranose 
and $(1 \rightarrow 3,6)$ - $\alpha$-D-glucosepyranose. The branches were composed of one $\rightarrow 1$ ) $\alpha$-D-lyxopyranose residue. The D-configuration monosaccharide of $\mathrm{AC}-1$ (Fig. 2) was in accordance with the GC-MS analysis (Fig. 6).

Structure elucidation of $A C-1$. On the basis of the above experimental data, it was elucidated that the possible structure of AC-1 had a backbone of 1,4-linked D-glucose and 1,3,6-linked D-glucose with branches predominantly composed of one 1-linked D-lyxose residue (Fig. 7).

DPPH free radical scavenging activity of $A C-1 . \mathrm{DPPH}$ radicals have an odd (unpaired) number of electrons and a strong absorption at $517 \mathrm{~nm}$. When a radical scavenger pairs to an unpaired electron, the strong absorption is gradually decreased. It is visually noticeable as a change in color from purple to yellow. Amanita caesarea exhibited a comparable anti-oxidant activity with that of standard Vc at varying concentrations tested. There was a dose-dependent increase in the percentage antioxidant activity (Fig. 8). The extract of AC-1 at a concentration of $1.0 \mathrm{mg} / \mathrm{ml}$ showed $37.09 \%$ free radical scavenging activity and $89.74 \%$ at $6.0 \mathrm{mg} / \mathrm{ml}$. The results showed that the $\mathrm{IC}_{50}$ value of eliminating $\mathrm{DPPH}^{-}$radicals was $\sim 1.69 \mathrm{mg} / \mathrm{ml}$ for $\mathrm{AC}-1$, which indicated that $\mathrm{AC}-1$ has a significant effect on the scavenging $\mathrm{DPPH}^{-}$radical, particularly at a high concentration. However, the scavenging ability was lower than that of $\mathrm{V}_{\mathrm{c}}$. There was no significant difference between the DPPH free radical scavenging activity when the concentration of AC-1 was $6 \mathrm{mg} / \mathrm{ml}$ compared with the $\mathrm{Vc}$ and BHT.

ABTS radical scavenging activity of $A C-1$. The $\mathrm{ABTS}^{+}$radical scavenging activity of AC-1 was measured spectrophotometrically at $734 \mathrm{~nm}$. The results of antioxidant activity of AC-1 are shown in Fig. 9. It demonstrated that the absorbance of the $\mathrm{ABTS}^{+}$radical cation was decreased dose dependently, upon interaction with various concentrations of the extract, and the $\mathrm{IC}_{50}$ value of $\mathrm{AC}-1$ was $0.92 \mathrm{mg} / \mathrm{ml}$. Notably, there was no significant difference between ABTS free radical scavenging activity when the concentration of $\mathrm{AC}-1$ was $6-8 \mathrm{mg} / \mathrm{ml}$ compared with Vc.

\section{Discussion}

The present study reveals that the polysaccharide obtained from the fruiting bodies of Amanita caesarea, termed AC-1, is a heteropolysaccharide. The purified polysaccharide was confirmed to be of high purity. Structural analysis using GC-MS indicated that AC- 1 consists of $\alpha$-D-glucose and $\alpha$-D-lyxose at a ratio of 2:1. The backbone of AC-1 is composed of 1,4-linked $\alpha$-D-glucose and 1,3, 6-linked $\alpha$-D-glucose. The branches were predominantly composed of one 1-linked $\alpha$-D-lyxose residue.

Free radicals are atoms or groups of atoms with an odd (unpaired) number of electrons and are formed when oxygen interacts with certain molecules. Once formed these highly reactive radicals can initiate a chain reaction. Damage is caused when free radicals react with important cellular components, such as DNA or the cell membrane. Cells may function poorly or die if this occurs $(21,22)$. Antioxidants are molecules which can safely interact with free radicals and terminate the chain reaction before vital molecules are damaged. Antioxidants are also thought to serve a role in slowing the aging process, and preventing heart disease and stroke $(23,24)$. A large number of studies have demonstrated that fungal polysaccharides exhibit antioxidative activities and have low toxicity.

In the present study, DPPH free radical scavenging activity was analyzed and compared with that of Vc. It was demonstrated under the same experimental conditions, that the $\mathrm{IC}_{50}$ value of $\mathrm{Vc}$ was $0.1 \mathrm{mg} / \mathrm{ml}$ while the $\mathrm{IC}_{50}$ value of AC-1 was $1.69 \mathrm{mg} / \mathrm{ml}$. According to the respective structural characteristics of the polysaccharide molecules, we can infer that the weight-average molecular weight and the degree of polymerization of polysaccharide extracted are greater, while the amount of isolated hydroxyl are less. Therefore, the scavenging activity of AC-1 through the direct reduction of the electron and proton depend on the isolated hydroxyl, which make it possible to decease the capacity of the $\mathrm{N}=\mathrm{N}$ double bond in DPPH by oxidation- reduction reaction. In DPPH scavenging assays, the fraction of AC-1 extract exhibit the great antioxidant activity, which may be due to the amount of isolated hydroxyl.

Furthermore, in the ABTS radical scavenging activity experiment, different polysaccharide doses resulted in different scavenging ability of $\mathrm{ABTS}^{+}$. Under the experimental conditions of the ABTS radical scavenging activity assays, the $\mathrm{IC}_{50}$ value of $\mathrm{AC}-1$ is $0.92 \mathrm{mg} / \mathrm{ml}$. Overall the fruiting bodies of Amanita caesarea may be used in nutritional or pharmaceutical fields.

\section{Acknowledgements}

This project was supported by the National Natural Science Foundation of China (grant nos. 31400016 and 31200012); the Application Foundation Project of Sichuan Province (grant no. 2013JY0094); the Science and Technology Support Project of Sichuan Province (grant nos. 2014SZ0020 and 2014FZ0024), the Cultivate Major Projects of Sichuan Province (grant no. 14CZ0016); and the Open Foundation of Microbial Resources and Drug Development of Key Laboratory and of Guizhou Province (grant no. GZMRD-2014-002).

\section{References}

1. Hibbett DS, Binder M, Bischoff JF, Blackwell M, Cannon PF, Eriksson OE, Huhndorf S, James T, Kirk PM, Lücking R, et al: A higher-level phylogenetic classification of the fungi. Mycol Res 111: 509-547, 2007.

2. Bertozzi CR and Kiessling LL: Chemical glycobioloy. Science 291: 2357-2364, 2001.

3. Bittencourt VC, Figueiredo RT, da Silva RB, Mourão-Sá DS, Fernandez PL, Sassaki GL, Mulloy B, Bozza MT and Barreto-Bergter E: An alpha-glucan of Pseudallescheria boydii is involved in fungal phagocytosis and toll-like receptor activation. J Biol Chem 281: 22614-22623, 2006.

4. Borchers AT, Stern JS, Hackman RM, Keen CL and Gershwin ME: Mushrooms, tumors, and immunity. Proc Soc Exp Biol Med 221: 281-293, 1999.

5. Rudd PM, Elliott T, Cresswell P, Wilson IA and Dwek RA: Glycosylation and the immune system. Science 291: 2370-2376, 2001.

6. Brown GD, Herre J, Williams DL, Willment JA, Marshall AS and Gordon S: Dectin-1 mediates the biological effects of beta-glucans. J Exp Med 197: 1119-1124, 2003. 
7. Angeli JP, Ribeiro LR, Gonzaga ML, Soares Sde A, Ricardo MP, Tsuboy MS, Stidl R, Knasmueller S, Linhares RE and Mantovani MS: Protective effects of beta-glucan extracted from Agaricus brasiliensis against chemically induced DNA damage in human lymphocytes. Cell Biol Toxicol 22: 285-291, 2006.

8. Cordero RJ, Frases S, Guimaräes AJ, Rivera J and Casadevall A: Evidence for branching in cryptococcal capsular polysaccharides and consequences on its biological activity. Mol Microbiol 79: 1101-1117, 2011.

9. Wang F, Hou Y, Ding X, Hou W, Song B, Wang T, Li J and Zeng Y: Structure elucidation and antioxidant effect of a polysaccharide from Lactarius camphoratum (Bull.) Fr. Int J Biol Macromol 62: 131-136, 2013.

10. Wu XM and Tu PF: Isolation and characterization of alpha-(1 $\rightarrow 6$ )-glucans from Cistanche deserticola. J Asian Nat Prod Res 7: 823-828, 2005.

11. Staub AM: Removal of protein-Sevag method. Carbohydr Chem 5: 5-6, 1965.

12. Kumar CG, Joo HS, Choi JW, Koo YM and Chang CS: Purification and characterization of extracellular polysaccharide from haloalkalophilic Bacillus sp. I-450. Enzyme Microb Technol 34: 673-681, 2004.

13. Pang XB, Yao WB, Yang XB, Xie C, Liu D, Zhang J and Gao XD: Purification, characterization and biological activity on hepatocytes of a polysaccharide from Flammulina velutipes mycelium. Carbohydr Res 70: 291-297, 2007.

14. Yu RM, Yin Y, Yang W, Ma WL, Yang L, Chen XJ, Zhang Z, Ye B and Song LY: Structural elucidation and biological activity of a novel polysaccharide by alkaline extraction from cultured Cordyceps militaris. Carbohydr Polym 75: 166-171, 2009.

15. Fan Y, He X, Zhou S, Luo A, He T and Chun Z: Composition analysis and antioxidant activity of polysaccharide from Dendrobium denneanum. Int J Biol Macromol 45: 169-173, 2009.
16. Sun Y and Liu J: Purification, structure and immunobiological activity of a water-soluble polysaccharide from the fruiting body of Pleurotus ostreatus. Bioresour Technol 100: 983-986, 2009.

17. Rongmin Y, Yin Y, Wei Yang, Weili M, Lin Y, Xiujuan C, Zhang Z, Bin Y and Liyan S: Structural elucidation and biological activity of a novel polysaccharide by alkaline extraction from cultured Cordyceps militaris. Carbohydr Polymers 75: 166-171, 2009.

18. Ding X, Hou YL and Hou WR: Structure elucidation and antioxidant activity of a novel polysaccharide isolated from Boletus speciosus Forst. Int J Biol Macromol 50: 613-618, 2012.

19. Chen J, Hu $\mathrm{T}$ and Zheng R: Antioxidant activities of Sophora subprosrate polysaccharide in immunosuppressed mice. Int Immunopharmacol 7: 547-553, 2007.

20. Luo DH: Identification of structure and antioxidant activity of a fraction of polysaccharide purified from Dioscorea nipponica Makino. Carbohydr Polym 71: 544-549, 2008.

21. Kumar V, Lemos M, Sharma M and Shriram V: Antioxidant and DNA damage protecting activities of Eulophia nuda Lindl. Free Radicals and Antioxidants 3: 55-60, 2013.

22. Blois MS: Antioxidant determination by use of stable free radicals. Nature 29: 1199-1200, 1958.

23. Hertog MGL, Feskens EJM, Kromhout D, Hertog MGL, Hollman PCH, Hertog MGL and Katan MB: Dietary antioxidant flavonoids and risk of coronary heart disease: The Zutphen Elderly Study. Lancet 342: 1007-1011, 1993.

24. Kushi LH, Folsom AR, Prineas RJ, Mink PJ, Wu Y and Bostick RM: Dietary antioxidant vitamins and death from coronary heart disease in postmenopausal women. New Engl J Med 334: 1156-1162, 1996. 